\title{
Are some physicians afraid of the COVID-19 vaccines? A tale of motivated reasoning
}

\section{Luis Claudio Lemos Correia' (1) João de Deus Barreto Segundo² (1)}

${ }^{1}$ Corresponding author. Escola Bahiana de Medicina e Saúde Pública (Salvador). Bahia, Brazil. luis.correia@bahiana.edu.br ${ }^{2}$ Escola Bahiana de Medicina e Saúde Pública (Salvador). Bahia, Brazil. jao.barreto@gmail.com

December 2020 was a special month as the world celebrated the approval of the first vaccine for COVID-19. The first of many vaccines that would allow for deploying pandemic control measures in 2021. Despite its rigorous approval process, along came an overbearing public discussion by physicians on efficacy and safety. Questions emerged on social media, television specials, and webinars, whose titles connoted the need for clarification as to if it were a confusing subject: "what we need to know," "benefits and risks," to mention some. There has been an excessive public concern with details as if those were more important than the fact that humanity could finally hope for the end of a humanitarian crisis entering its second year with a death toll in the millions. Vaccines have been around for at least 200 years. So, why the noise among physicians?

The development of multiple vaccines in such a short timeframe is one of the greatest achievements in biomedical technology research history: with enormous efficacy (immense effect size), unprecedented epidemiological usefulness, methodological rigor, compliance to ethical standards, outstanding international cooperation, and very low clinical cost (risk). Those aspects alone should have overshadowed details such as "how long the effect of vaccination would last," whether "immunized people would be capable of transmitting the disease" or "the vaccine reduced the severity of the disease," along with "concerns about the unprecedented nature of RNA-based technology" to point out a few.

We do not mean to convey that those are not important questions for the scientific community to discuss among themselves in the long run. Right now, however, there is an evident asymmetry between the rich level of evidence that we already have on the multiple vaccines' safety, usefulness, efficacy, and effectiveness as opposed to the interest in publicly discussing issues that are not life-and-death. Do these many doubts contaminate the perception of the public and cause vaccine hesitancy? That is a known unknown: the publicization of a given issue on media channels does not necessarily amount to persuasion nor behavior change, but it may result in cognitive fatigue, misinformation, and polarization ${ }^{1-3}$, which are undesirable as well, given the extent of the crisis. 
Typically, physicians overestimate the potential benefit of conduct and underestimate the damage. ${ }^{4}$ In the case of the COVID-19 vaccines, public debate among physicians seems to indicate some are underestimating the obvious benefit meanwhile overestimating outlandish harms. Thus, there is an evident asymmetry between the current exaggerated caution with details of the approved vaccines and the lack of caution when promoting popular medical treatments without proof of benefits, characterizing overuse. Unfortunately, the overuse phenomenon is no recent trend in medical practice and calls for a theoretical framework of its own for proper analysis and customized solutions., 4 So, is it ethical to foster such a public debate on secondary issues regarding vaccines at this present moment without accompanying reliable evidence to do so?

Cognition is prone to self-serving biases that allow for cherry-picking evidence that conforms to one's own worldview. ${ }^{3}$ To what extent cognition is inherently biased and how, on top of that, institutional/elite cues and ideology-rich contexts interact with mindsets has been open for debate for, at least, fifty years now.., 6 Since it has been posited that, in public debate, individuals who perceive their opinion to belong to a minority would commonly rather silence than expose themselves to rejection. ${ }^{?}$ Belonging to groups and, thus, to identities plays an important part in cognition and decision-making. ${ }^{3,6,7}$ Those phenomena appear to manifest even among physicians. 5

Such a debate on a vaccine, held in public, without proper evidence to inform it offers little to no benefit to policy-makers and society in general. It is rather unscientific, however, as it offers fertile grounds for misinformation stirring unnecessary polarization $1-3,6,7,7$ that may harm the only message that matters right now, the common message around which all physicians and the scientific community should band together during these next months: everyone must get vaccinated no matter what approved vaccine is available in their countries, as there is no possibility of ending the pandemic without vaccination. ${ }^{-}$By doing so, we not only acknowledge these remarkable scientific achievements of the past year, but we also avoid undermining public perception of the sanitary regulatory agencies behind the rigorous vetting of these new pieces of biomedical technology.
Vaccination is now a worldwide public health imperative. Deaths after vaccines' approval might as well be computed as avoidable deaths. So, in order to argue that questioning vaccine right now is not an efficient public debate, let us look at the available evidence for one of the vaccines with the highest efficacy and effect size.

\section{The COVID-19 vaccine endgame}

Uncertainty acknowledgment allows for the mastery of probabilistic thinking. However, uncertainty must not be mistaken for lack of confidence as it is modulated by the soundness of the evidence. 9 The Pfizer study with mRNA vaccine was a large clinical trial, with statistical power and sufficient precision to reject the null hypothesis with a low risk of type I error and high precision in estimating the effect size. There was no change in protocol nor primary outcome when the article is compared to the prior publication of its methodology. It was a randomized, doubleblind clinical trial in which the primary outcome was the occurrence of COVID-19 after the second dose, a feasible, useful, reliable outcome given the pandemic context. 10,11

One of the concerns that have been publicly raised is that there was no active placebo in the Pfizer ${ }^{10}$ nor in the Moderna study ${ }^{12}$, that is, the use of something in the control group that promoted similar adverse effects (such as the vaccine for another disease). An active placebo is important when treatment may cause adverse effects that can unmask the blinding. The patient ends up knowing that he was allocated to the vaccine group. However, the biggest problem with unmasked studies lies in the subjectivity of the outcome. The COVID-19 outcome gains objectivity to the extent that it needs laboratory confirmation. That would reduce the risk of bias, but it would not eliminate it completely, as patients who presumed they had not been vaccinated could have had reported more symptoms, be more tested, overestimating disease incidence. However, in the passive placebocontrolled study ${ }^{10}$, this mechanism is unlikely to justify the significant contrast between the two groups (168 cases versus 8 cases of COVID-19). Overestimation cannot explain such disparity. 
Another concern has been the modified intention to treat. Intention-to-treat analysis keeps all individuals in the group initially randomized, even if they did not receive the intervention they were allocated. This technique prevents the homogenizing effect of randomization from being lost, mainly because patients who violate the protocol have a different average risk. Modified intention-to-treat occurs when this rule is not fully observed, and some of the individuals are excluded from the analysis. It is a euphemism for analysis by protocol, which biases the study design in favor of the intervention.

In the Pfizer study 10 , three types of patients were excluded after randomization: those who violated some form of the protocol, those who had less than two months of follow-up, and the few who had infection between first and second doses, thus making it impossible to assess effectiveness. Therefore, of the total of 43,500 randomized patients, 37,700 were analyzed. Around $14 \%$ of randomized patients were left out, a number compatible with the usual crossover in interventional studies. Usually, a protocol analysis or modified intention-to-treat in this situation would be considered to be at high risk of bias. However, vaccine studies have two particularities that make analysis by protocol adequate. First, a vaccine is a universal approach. It is not selected as an intervention by the physician based on the patient's condition, and it is applied to people without the disease. The protocol violation does not occur due to a change in conduct based on the patient's condition; it is much more of an unbiased stochastic (random!) event. That reduces the confounding effect created by exclusion after randomization.

Vaccines usually have a huge effect size, different from usual treatments. Thus, computing people who have not used a vaccine as if they had (and vice versa) would bring about a major change in the outcome. Moreover, that would amount to a bigger impact than the eventual confounding effect of the analysis by protocol. Vaccination studies represent one of those situations in which analysis by protocol gains a level of evidence similar to an analysis by intentionto-treat. Consequently, the appraised study displays confirmatory, reliable evidence, with a low risk of bias and random error. ${ }^{10,11}$

\section{Paradigm shift}

The usual number-needed-to-treat (NNT) derives from the individual likelihood that a subject who receives treatment will benefit from it. This likelihood is the absolute reduction in risk. A person's individual risk of acquiring COVID-19 is not high: the whopping 7 million cases in Brazil by December 2020 promoted an individual probability of that event occurring at $3 \%$. In the Pfizer study, the incidence of the disease in the placebo group was much lower, $0.88 \% .10$ So, although the relative risk reduction is huge, the absolute risk reduction was only $0.84 \%$ (0.88 - 0.04), which results in an NNT as high as 119 . Considering the intervention (vaccine) for a single patient, it would not be an impressive course of treatment. However, in the absence of physical distancing measures, a sick person is estimated to have the potential to contaminate three others $(\mathrm{RO}=3)$. Thus, those 119 would prevent not only 1 case but 4, reducing the NNT to 30 . That is a systemic rationale to be adequately deployed to understanding vaccination during the pandemic with an exponential effect the following suit. The vaccine is not an intervention for a group of patients, and it is for a single patient: the population. When the intervention is deployed, it impacts the NNT, which would be 1 if $70 \%$ of the people are vaccinated, ending the pandemic.

At an individual level, that rationale works as follows. The primary analysis in the Pfizer trial was by personyear (hazard), but for simplicity, the percentage of infected (risk) is described here: in the vaccine group, the incidence of COVID-19 was $0.04 \%$ (8 cases), compared to $0.88 \%$ (162 cases) in the placebo group. That represents a relative risk of 0.05 (almost null), which translates into a 95\% relative risk reduction, with a precise confidence interval $(90 \%-98 \%) \cdot .^{10}$ This enormous effect size almost eliminates the unwanted outcome, which is very rare in disease treatments, whose relative risk reduction sits well below $30 \%$. So, this magnitude of benefit is not surprising, nor is it too good to be true. 


\section{The ethics (or lack thereof) of cherry- picking concerns}

Another unnecessary discussion that has emerged is whether vaccines reduce mortality ${ }^{13}$ since the outcomes analyzed by the clinical trials were not death. This question disregards the likeliness of the fatal outcome, a principle applicable to preventing the onset of diseases that lead some people to death. We are talking about an intervention that reduces by $95 \%$ the incidence of disease ${ }^{10}$ that has already killed 2.8 million people in the world. ${ }^{14}$ COVID-19 deaths are rare, but they do exist. If COVID-19 kills sometimes and COVID-19 is eliminated preemptively, there will be death prevention as well. It is tautological.

In cases of disease prevention that kill and regarding a great asymmetry between potential risk and benefit, there is no need for the primary outcome to be mortality. The proof of effectiveness is in reducing the disease; otherwise, it would be a waste in research when good, rigorous, feasible, ethical research is needed the most. ${ }^{15-11}$ Not to mention that, as death is a rare outcome in the disease, the change in the outcome of the clinical trial would require a much bigger sample size, most frequent follow-up, increasing random error probability and thus making the trials lengthier and expensive ${ }^{13}$, meanwhile the crisis would still be claiming lives.

After recovery, the lasting effects of COVID-19 are a known unknown, and the emerging evidence paints a dire picture. $\frac{18}{}$ It will be years before life, and social scientists manage to assess the impact of COVID-19 not only in the human body but in household dynamics, gender, and racial inequality, workforce, and social security as some who recovered are expected not to be able to be fully reintegrated into the workforce. So, a lengthy trial assessing a tautological outcome would not only face loss in feasibility but would also be unethical. Bottom line: questioning vaccine efficacy on the premise of the absence of hard outcome may also amount to an unethical shallow debate. Unfortunately, that is not the only entry in cherrypicking concerns on this ongoing public lengthy and fruitless debate on vaccine efficacy. "New technology in the case of the RNA vaccine," "short-term followup measured in months," "highly selected population sample in the clinical trials," to mention some of the other voiced concerns regarding vaccine safety.
Meanwhile, there is a huge asymmetry between potential effectiveness and safety: a total absence of serious adverse events in 18,000 patients who received the vaccine. What is to be doubted exactly? And to what end?

Doubts about the vaccination's effectiveness in younger individuals and safety on people with a history of allergy have also arisen. However, in all clinical trials of efficacy and safety, concepts have consistently been demonstrated in hyper-selected samples in favor of the study's internal validity, which can be extrapolated to the general population based on the rarity of the interaction phenomenon, which justifies the principle of complacency. In case of evidence of high internal validity in analytical studies (those which test hypotheses), the external validity may be safely extrapolated beyond the characteristics of the studied sample. Therefore, the mentioned safety concerns are prejudiced against the most vulnerable, who did not participate in the vaccine trials. There is no need for a subgroup analysis proving vaccine effectiveness in different types of people as those analyses suffer a high risk of random error and a different result from the general study has, by default, a lower predictive value.

At this point, we come to an intriguing question: why regarding the vaccines, is there such reluctance opposing the soundness of the evidence?

\section{Motivated reasoning}

As health professionals, we are predisposed to overuse. Physicians tend to overestimate the benefits of their therapies and the accuracy of their tests, underestimating the risks, which may amount to selfserving biases., 5 Humans are averse to uncertainty, and among physicians, it is no different. 5,9 That may lead to non-evidence-based treatments ${ }^{19}$, which may be based on heuristics ${ }^{5,20}$ structured by motivated reasoning, a cognitive predisposition to satisfy the need to belong to a particular group/identity at the expense of rationality. ${ }^{3,6}$ Within the model of identityprotective cognition, belief polarization could be explained as a process of motivated reasoning driven by the individual psychological need to maintain 
beliefs that preserve their status in the groups they are affiliated to. ${ }^{3,6}$ as a tacit sanction of publicly going against group ideology may be ostracism. ${ }^{7}$ The cost of changing opinions is measured thus in predicted social gains and losses, which may not be an active, reflexive process of reasoning. $3,6,7,1$

Independent studies also indicate it is unlikely for cherry-picking evidence to be exclusively ideologically motivated, as ideology appears to play out as an aggravating factor within a general framework of motivated reasoning complexity, which may have intrinsic components to it as well. $-\frac{3,21}{21}$ Apart from the lack of evidence, pinpointing partisanship alone as a key factor in cherry-picking evidence towards vaccine technology would also reduce the problem to the momentary polarization present in countries such as Brazil, and motivated reasoning regarding health decision-making precedes the current crisis as the literature in overuse has been showing time and again. $-3-5$

However, the precise interplay between internal biases and external context has been eluding scientists for many years. .6 Although scientific training may help reduce the influence of partisanship, religion, and other forms of identarian affiliations that offer worldview heuristics, it does so to a limited effect. It is suspected that some heuristics are too essential to one's identity to be discredited by the individual even despite their scientific training. ${ }^{19-22}$

\section{Present Bias}

So, if not ideological heuristics alone, what else could play a role in motivating reasoning against vaccine technology? Why risk aversion (of dying from COVID-19) and the perceived desire for security do not prevail in the long run? Could other biases be at play among physicians, such as the present bias?

It is easier to persuade someone to adhere to measures of self-preservation in situations of imminent danger. ${ }^{22-24}$ Hardly anyone would question the need for an oxygen mask in an airplane with a depressurized cabin, but some question the need for face masks to prevent spreading COVID-19 despite physical barriers beingself-evidentfor at leastreducing transmission. Both situations may cause suffocation. Note that the risk perception in a depressurized cabin is different from the one involved in one presuming to, in the future, developing severe COVID-19 (or any other disease). The disease is possible incident weeks ahead in the future, while a depressurized cabin may signal death minutes ahead in the future. The difficulty in adhering to preventive health measures associated with lifestyle changes is fairly explained by our tendency to prefer immediate gratification: the present bias. ${ }^{24}$ It may even manifest as a conflict between short-term self-interest opposing longerterm collective interest ${ }^{23}$ or cherry-picking news that best serves an already present worldview as not undergo isolation in the future. $2,3, ., 7$

In the case of COVID-19, we may perceive that, individually, our risk is neither high nor imminent. The vast majority of people we know do not die from COVID-19 as its lethality is low. Death by COVID-19 thus remains not in the here and now but in a future that may not come to pass. Therefore, risk aversion for COVID-19 is not strong enough, and this could make room for the present bias to manifest. $22-25$ Nevertheless, what could be so rewarding about the present for some physicians to remain stuck in the pre-COVID19-vaccine timeline?

For one, media exposure is associated with more citations $2,26-28$ which are currency and proxy for reputation in the academe. $29-31$ Non-academic physicians may also stand to gain from media exposure. In their case, while their social gain would not be materialized in the form of citation, gains could manifest in strengthening in-group affiliations $3,6,2,2,23,25$ to avoid social losses ${ }^{3,6,7}$ utilizing broadcasting their beliefs/worldviews. In a polarized public arena, divergent opinions may not be as prone to public sanction as they would in a non-polarized public arena dominated by a majoritarian worldview. In a polarized public arena, worldviews could be competing in order for the winning worldview to define facts and frame solutions. Consequently, all competing worldviews would be expected to make as much noise as possible to silence dissent. ${ }^{7}$ However, they may result in misinformation or disinformation due to motivated reasoning among the audience. $1-3,-6,21-23,25$ 
Evidence also shows that threats perceived to be overwhelming send individuals further into motivated reasoning due to the perceived inability to solve the issues at hand, opening a window for the present bias to manifest and enlarging opinion/behavioral gulfs between out-groups. $3,6,2,2,23,25$ Then, as short-term gratifications may be perceived to be more important than long-term rewards $s^{22-25}$, feeling safe within one's identity and in-group $3,6,22,23,25$ as to avoid isolation ${ }^{7}$ could be preferred. Isolation is usually associated with poorer health outcomes and stress, confusion, anger, and violence ${ }^{23}$ and nobody wants that. In the pandemic, perceived alienation from in-group may be automatically perceived as the real big threat to survival due to the complex interplay between inherent cognitive biases and context. One must remember that human survival once depended strictly on homophilic cooperation, that is, the preference to cooperate with perceived in-groups at the expense of out-groups (which is also the root of xenophobia, racism, homophobia, and others). Nevertheless, that does not mean people cannot cooperate in a crisis. Evidence shows quite the opposite, that people can and usually cooperate during emergencies but not within polarized environments, which usually feeds further polarization. $22,23,25$

\section{Cascade}

More media exposure does not induce more persuasion nor decision-making in a perfect controlled reaction of cascading events., ${ }^{1,2}$ Slight gain in reputation is to be expected to the mouthpieces trumpeting this or that non-evidence-based solution during the pandemic as motivated reasoning is also to be expected from audience members. 23.6 We are all preaching to the already converted in our echo chambers. ${ }^{2.3}$ However, silencing is not an ethical option $^{32}$, as refraining from evidence-based public debate right now may allow for non-evidence-based arguments to prevail and set the public agenda. ? $^{-}$ However, caution is advised. The evidence-based way to engage in such a debate is by bringing the evidence along with the context it was generated in? framed as to appeal to our shared humanity across the identarian gulfs $\mathrm{s}^{22,23,25}$, thus promoting scientific training 21 and avoiding pitfalls such as crystallized perceptions of the public regarding scientists or politicians. ${ }^{-1}$ Physicians and scientists cannot be complicit in polarization by evading these issues. Social communication must also be evidence-based.

As worldwide vaccination progresses and the perceived existential threat is expected to fade in people's hearts and minds, pressure on cognition and social bonds may be alleviated, making pandemic polemics less and less attractive in the mainstream public arena and causing them to be pushed back into science fiction. Evidence suggests divisive leadership and harmful actions tend to be judged by public opinion more harshly than harmful inactions. $\underline{\underline{23}}$ What is to happen to those who are now stirring polemics and polarization for personal gain ${ }^{2}$ once, soon, the majority decides that those behaviors should be considered harmful action and push said individuals into public opinion limbo?

As for us, we must motivate [ly] focus on the actual important issue at hand: multiple vaccines have been rolled out, and we must get everyone vaccinated as soon as possible with the approved vaccines made available to each and every individual in the world. And what a luxury it would be if only every individual could be granted access to the best vaccine technologies currently available. If only we could cooperate that far.

\section{Competing interests}

No financial, legal, or political competing interests with third parties (government, commercial, private foundation, etc.) were disclosed for any aspect of the submitted work (including but not limited to grants, data monitoring board, study design, manuscript preparation, statistical analysis, etc.).

\section{References}

1. Farjam M, Bianchi F, Squazzoni F, Bravo G. Dangerous liaisons: an online experiment on the role of scientific experts and politicians in ensuring public support for anti-COVID measures. R Soc Open Sci. 2021;8(3):201310. http://doi.org/10.1098/ rsos.201310 
2. Alves CPL, Barreto Segundo JD, Costa GG, Pereira-Cenci T, Lima KC, Demarco FF, et al. Show me the study! Science by press release is not science 2021. http://dx.doi.org/10.31222/osf.io/ tk8w9

3. Williams D. Motivated ignorance, rationality, and democratic politics. Synthese. 2020:1-21. https://doi.org/10.1007/s11229-02002549-8

4. Alencar Neto J, Farina E, Sampaio MCN. Schrödinger's Cat Bias: A New Cognitive Bias Proposed. Cureus. 2021;13(1):e12697. https://dx.doi.org/10.7759\%2Fcureus.12697

5. Djulbegovic B, Elqayam S, Dale W. Rational decision making in medicine: Implications for overuse and underuse. J Eval Clin Pract. 2018;24(3):655-65. https://doi.org/10.1111/jep.12851

6. Rekker R. The nature and origins of political polarization over science. Public Underst Sci. 2021;30(4):352-68. https://doi. org/10.1177/0963662521989193

7. Noelle-Neumann E. The spiral of silence a theory of public opinion. J Commun. 1974;24(2):43-51. https://doi. org/10.1111/j.1460-2466.1974.tb00367.x

8. Chaves A. What is the evidence in favor of herd immunity for the COVID-19 pandemics? J Evid Based Healthc. 2020;2(2). http:// dx.doi.org/10.17267/2675-021Xevidence.v2i2.3335

9. Bobbio M. In praise of "Our stubborn quest for diagnostic certainty". J Evid Based Healthc. 2019;1(2):106-11. http://dx. doi. org/10.17267/2675-021Xevidence.v1i2.2625

10. Polack FP, Thomas SJ, Kitchin N, Absalon J, Gurtman A, Lockhart S, et al. Safety and Efficacy of the BNT162b2 mRNA Covid-19 Vaccine. N Engl J Med. 2020;383:2603-15. http://dx.doi. org/10.1056/NEJMoa2034577

11. Clinical Trials.gov. Study to Describe the Safety, Tolerability, Immunogenicity, and Efficacy of RNA Vaccine Candidates Against COVID-19 in Healthy Individuals [Internet]. U.S National Library of Medicine. [cited 2021 Mar. 28]. Available from: https:// clinicaltrials.gov/ct2/show/NCT04368728

12. Baden LR, El Sahly HM, Essink B, Kotloff K, Frey S, Novak R, et al. Efficacy and Safety of the mRNA-1273 SARS-CoV-2 Vaccine. N Engl J Med 2021;384:403-16. http://dx.doi.org/10.1056/ NEJMoa2035389

13. Doshi P. Will covid-19 vaccines save lives? Current trials aren't designed to tell us. BMJ. 2020;371:m4037. https://doi.org/10.1136/ bmj.m4037

14. Coronavirus Resource Center. COVID-19 Dashboard by the Center for Systems Science and Engineering (CSSE) at Johns Hopkins University (JHU) [Internet]. Johns Hopkins University of Medicine [cited 2021 Apr. 3]. Available from: https://coronavirus. jhu.edu/map.html
15. Glasziou PP, Sanders S, Hoffmann T. Waste in covid-19 research. BMJ. 2020;369:m1847. https://doi.org/10.1136/bmj. m1847

16. Janiaud P, Axfors C, Van't Hooft J, Saccilotto R, Agarwal A, Appenzeller-Herzog C, et al. The worldwide clinical trial research response to the COVID-19 pandemic - the first 100 days. F1000Res. 2020;9:1193. https://doi.org/10.12688/ f1000research.26707.2

17. Correia L, Barreto Segundo JD. An immunization program against the COVID-19 infodemic. J Evid Based Healthc. 2020;2(1):79. http://dx.doi.org/10.17267/2675-021Xevidence.v2i1.3124

18. Al-Aly Z, Xie Y, Bowe B. High-dimensional characterization of post-acute sequalae of COVID-19. Nature. 2021. https://doi. org/10.1038/s41586-021-03553-9

19. Correia L, Lopes J, Garcez F, Campion E, Barcellos G, BarretoFilho J. Physicians' preference towards the non-evidence based hydroxychloroquine treatment for COVID-19: the pandemic effect. J Evid Based Healthc. 2020;2(1):10-15. http://dx.doi. org/10.17267/2675-021Xevidence.v2i1.3014

20. Solla D. Hydroxychloroquine for the treatment of COVID-19: an approach based on the philosophy of science and heuristics. J Evid Based Healthc. 2020;2(1):28-31. http://dx.doi.org/10.17267/2675021Xevidence.v2i1.2890

21. Weisberg DS, Landrum AR, Hamilton J, Weisberg M. Knowledge about the nature of science increases public acceptance of science regardless of identity factors. Public Underst Sci. 2020;30(2):12038. https://doi.org/10.1177/0963662520977700

22. West R, Michie S, Rubin GJ, Amlôt R. Applying principles of behaviour change to reduce SARS-CoV-2 transmission. Nat Hum Behav. 2020;4:451-9. https://doi.org/10.1038/s41562-020-0887-9

23. Bavel JJV, Baicker K, Boggio PS, Capraro V, Cichocka A, Cikara $M$, et al. Using social and behavioural science to support COVID-19 pandemic response. Nat Hum Behav. 2020;4(5):460-71. https:// doi.org/10.1038/s41562-020-0884-z

24. Wang Y, Sloan FA. Present bias and health. J Risk Uncertain. 2018;57(2):177-98. https://doi.org/10.1007/s11166-018-9289-z

25. Bendixen T. How cultural evolution can inform the science of science communication-and vice versa. Humanit Soc Sci Commun. 2020;7(1):135. https://doi.org/10.1057/s41599-02000634-4

26. Anderson PS, Odom AR, Gray HM, Jones JB, Christensen WF, Hollingshead T, et al. A case study exploring associations between popular media attention of scientific research and scientific citations. PLoS One. 2020;15(7):e0234912. https://dx.doi. org/10.1371\%2Fjournal.pone.0234912 
27. Dumas-Mallet E, Garenne A, Boraud T, Gonon F. Does newspapers coverage influence the citations count of scientific publications? An analysis of biomedical studies. Scientometrics. 2020;123(1):413-27. http://dx.doi.org/10.1007/s11192-020-033801

28. Fanelli D. Any publicity is better than none: newspaper coverage increases citations, in the UK more than in Italy. Scientometrics. 2013;95(3):1167-77. http://dx.doi.org/10.1007/ s11192-012-0925-0

29. Hirsch JE. Superconductivity, What the H? The Emperor Has No Clothes. Physics and Society [Internet]. 2020;49(1):4-9. Available from: https://arxiv.org/ftp/arxiv/papers/2001/2001.09496.pdf
30. Génova G, Astudillo H, Fraga A. The Scientometric Bubble Considered Harmful. Sci Eng Ethics. 2016;22(1):227-35. https:// doi.org/10.1007/s11948-015-9632-6

31. McKiernan EC, Schimanski LA, Nieves CM, Matthias L, Niles MT, Alperin JP. Use of the Journal Impact Factor in academic review, promotion, and tenure evaluations. Elife. 2019;8:e47338. https:// dx.doi.org/10.7554\%2FeLife.47338

32. Barreto Segundo JD, Villalobos APO, Correia LC. (2019). Science must speak truth to power. J Evid Based Healthc. 2019;1(1):10-4. http://dx.doi.org/10.17267/2675-021Xevidence.v111.2366 